\title{
Spatial language influences memory for spatial scenes
}

\author{
Michele I. Feist \\ University of Louisiana, Lafayette, Louisiana \\ AND \\ DEDRE GENTNER \\ Northwestern University, Evanston, Illinois
}

\begin{abstract}
Does language influence recognition for spatial scenes? In Experiments 1 and 2, participants viewed ambiguous pictures, with or without spatial sentences. In a yes-no recognition task, only the spatial sentences group made more false alarms toward the center of the spatial category than in the other direction; three other comparison groups showed no such tendency. This shift toward the core of the semantic category suggests that spatial language interacted with perceptual information during encoding. In Experiment 3, we varied the materials to test the interactive encoding account against a separate encoding account in which separately stored sentences are accessed during picture recognition. The results support the interactive encoding account in which spatial language influences the encoding and memory of spatial relations.
\end{abstract}

In recent times, there has been a resurgence of interest in the question of whether and how language influences thought (Bowerman \& Levinson, 2001; Gentner \& Goldin-Meadow, 2003; Gumperz \& Levinson, 1996). Some of this work is in the classic tradition of the strong version of the language-and-thought hypothesis, linguistic determinism: the thesis that the language a person speaks determines the way in which one perceives and encodes the world. This version is expressed in Whorf's (1956) quote of Sapir: "[w]e see and hear and otherwise experience very largely as we do because the language habits of our community predispose certain choices of interpretation" (p. 134; Sapir, 1929, p. 210). For example, in one influential line of research, Levinson and his colleagues (Levinson, 1996, 2003; Levinson, Kita, Haun, \& Rasch, 2002; Pederson et al., 1998) have suggested that the spatial frames of reference employed by a given language influence its speakers' performance on nonlinguistic tasks. They report that speakers of languages that predominantly use egocentric spatial terms (such as left-right in English) attend greatly to the left-right order of objects with respect to the speaker. In contrast, speakers of languages (e.g., Tzeltál) that predominantly use absolute spatial terms (the equivalent of north-south) encode positions with respect to a global framework. Levinson et al.'s (2002) conclusion that performance in simple nonlinguistic tasks reflects the dominant spatial frame in a participant's language has generated considerable interest as well as some challenges. Li and Gleitman (2002) argued that an array of strategies can also be induced in monolingual English speakers by changing the task context, to which Levinson et al. (2002) countered that within-language context effects are not inconsistent with substantial crosslinguistic differences in default patterns of usage (see also Majid, Bowerman, Kita, Haun, \& Levinson, 2004). Such within-language effects have been reported by Taylor and Tversky (1996), who found that changes in aspects of the scene to be described resulted in changes in English speakers' choice of linguistic frame of reference (perspective, in Taylor and Tversky's terms). Other investigations of the strong Whorfian hypothesis have likewise generated a mix of positive findings (e.g., Boroditsky, 2001; Lucy, 1992) and negative findings (e.g., Gennari, Sloman, Malt, \& Fitch, 2002; Malt, Sloman, \& Gennari, 2003; Papafragou, Massey, \& Gleitman, 2002).

The debate over strong versions of the Whorfian hypothesis, as exemplified above, may require considerable research before it can be settled. However, current discussions have also refined and differentiated the languageand-thought question (Bowerman \& Levinson, 2001; Gentner \& Goldin-Meadow, 2003). One moderate version is that habitual ways of talking and the construals habitually made for linguistic purposes become especially available in nonlinguistic contexts (Hunt \& Agnoli, 1991). A still weaker version is Slobin's $(1996,2003)$ thinkingfor-speaking hypothesis, which states that linguistic influences occur when language is used during a task (see also Pinker, 1989). The idea is that, in speaking, we are induced by the language we use to attend to certain aspects of the world while disregarding or de-emphasizing others.

M. I. Feist, feist@louisiana.edu 
Our interest here was in Slobin's thinking-for-speaking hypothesis. This view may seem unremarkable, but the fact that we spend so much of our time using language (both when communicating with others and when thinking within ourselves) suggests that such an influence may in fact be quite pervasive. In addition, this version of the hypothesis has the attractive feature that it permits testing for an effect of language on thought within a language, allowing researchers to factor out effects of culture that can muddy the interpretation of crosslinguistic studies.

In a number of studies, effects of language on cognitive measures when language was used during the task have been reported. For example, Billman and Krych (1998) found that hearing path verbs (e.g., enter) or manner verbs (e.g., walk) while watching videotaped motion events influenced English-speaking subjects' subsequent recognition of variants of the events: They were more likely to notice a change in manner if they had heard a manner verb, and likewise for path. Gentner and Loftus (1979) found that performance on a picture recognition task was influenced by having matched the picture to a verb 1 week prior to the recognition test. Participants who had matched a specific verb (e.g., hiking) to a general picture (a woman walking) were highly likely to choose the specific picture (a woman hiking) over the general picture they had actually received. The results of these studies suggest that attending to language during the encoding of scenes can influence scene encoding and subsequent recognition. Furthermore, these effects are not limited to motion verbs. Loewenstein and Gentner $(2001,2005)$ found that preschool children performed better in a difficult spatial mapping task when they had previously heard spatial terms describing the arrays. Hermer-Vazquez and her colleagues (Hermer-Vazquez, Moffet, \& Munkholm, 2001; Hermer-Vazquez, Spelke, \& Katsnelson, 1999) found that performance on a difficult spatial retrieval task was correlated with the ability to use the spatial language relevant to the task (but see Cheng \& Newcombe, 2005, and Learmonth, Nadel, \& Newcombe, 2002). Such findings suggest a role for spatial relational language in supporting spatial cognition.

In the present research, we asked whether the presence of spatial language-specifically, English spatial prepositions - can influence the encoding and memory of pictorial scenes. In answering this question, we sought evidence bearing on two questions: (1) whether participants' memories showed language-related alterations (Experiments 1 and 2), and (2) whether these alterations resulted from an active comparison of language and the picture in pursuit of a common construal of the two, a process we refer to as interactive encoding (Experiment 3 ).

We chose spatial prepositions for several reasons. First, spatial prepositions themselves have been the focus of much recent research (e.g., Bowerman \& Pederson, 1992, 1996; Coventry, Carmichael, \& Garrod, 1994; Coventry \& Garrod, 2004; Feist, 2000; Levinson, Meira, \& the Language and Cognition Group, 2003; Pederson et al., 1998; Regier, 1996; Vandeloise, 1991). Second, spatial prepositions are used with very high frequency even in nonspatial domains because space is a base domain for many metaphors (e.g., Boroditsky, 2001; Gentner \& Imai,
1992; Gentner, Imai, \& Boroditsky, 2002). Third, there has been comparatively little work on the possible effects of prepositions on the encoding of static spatial relations to date. Yet, spatial prepositions exhibit considerable variability across languages (Bowerman \& Pederson, 1992, 1996; Feist, 2000; Levinson et al., 2003). Coupled with evidence of very early learning (Bowerman \& Choi, 2003; Choi \& Bowerman, 1991), this variability raises the possibility that the semantic encodings in spatial prepositions might be especially likely to influence speakers' construals of the world.

\section{Design of the Experiments}

The basic plan was as follows. For each of the prepositions tested, we created a sentence (e.g., "The block is on the building") and a triad of pictures that varied in how well they fit the sentence (see Figure 1). The standard pictures were designed to be possible borderline exemplars of the spatial term, but not to be central exemplars. (In Norming Study 2B, we used spontaneous verbal descriptions to confirm that the standards did not by themselves give rise to the key prepositions.) For each standard, there were two variants, one of which (the plus variant) was a better exemplar of the spatial term, and one of which (the minus variant) was a poorer exemplar. Thus, the standard was designed so that the spatial preposition could apply to it, and the two variants were either more typical of the core prepositional category or less so. The participants were shown the set of standards and later were asked to recognize which picture they saw.

If participants apply the semantic categories of the spatial terms when encoding the standard, they should be more likely to have false alarms (FAs) to the plus variant than to the minus variant; that is, they should show a shift in recognition toward the category's center. Across three experiments, we varied the input conditions and asked under what conditions participants would show this effect. If we were to see language effects only when the participants were provided with language at encoding, this would provide support for a thinking-for-language hypothesis - a generalization of Slobin's thinking-forspeaking hypothesis to encompass comprehending as well as producing language. If, on the other hand, we were to see spatial category effects even without the presentation of language at encoding, this would support the possibility that language influences cognition in a more far-reaching manner.

The goal of the first two experiments was to test whether the presence of spatial language during encoding influences picture recognition. The aim of Experiment 3 was to extend the scope of the findings, as well as to clarify the explanation. We tested whether picture encoding can be influenced not just according to the specific terms but according to the dimensions on which the terms operate. In other words, instead of asking whether the use of on will shift the encoding of an ambiguous picture toward the center of the category named by on, in Experiment 3, we asked whether the use of any spatial term would highlight the spatial semantic system, shifting the encoding of all ambiguous pictures toward the centers of the nearest spatial semantic categories. The 
Plus variant
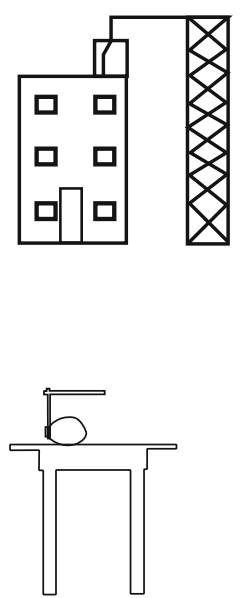

Standard

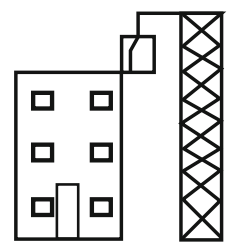

Minus variant

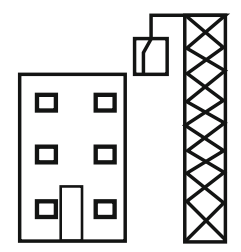

The block is on the building.
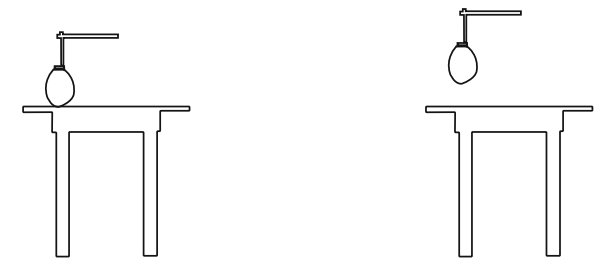

The balloon is on the table.

Figure 1. Example picture triads together with the sentence used for each triad. In each case, the plus variant was closer than the standard to the center of the spatial category conveyed by the sentence and the minus variant was farther.

study also allowed us to test whether our findings are explainable by intrusions from a separate encoding of the language used in the task, instead of by an interaction between language and the pictures (as we propose).

\section{EXPERIMENT 1}

In Experiment 1, we addressed the question of whether the presence of spatial language at encoding would influence recognition memory for simple spatial scenes. The participants viewed pictures depicting static spatial relations (e.g., a marionette standing on a table, or a coin in the palm of a hand). After participating in a 10-min distractor task, the participants performed a recognition task that included the original pictures and two variants.

In Experiment 1A, half the participants received two sentences along with each picture during the study portion and chose which best described the picture; the other half of the participants received only the pictures. One sentence (the target sentence) was plausible, and the other (the distractor sentence) was designed to be obviously wrong; its purpose was simply to cause the participants to read the correct sentence and encode the target spatial relational term. To forestall inducing a strategy of focusing directly on the preposition, only the nouns were changed in the distractor sentences. Thus, for the target sentence "The puppet is on the table," the distractor sentence was "The computer is on the desk." In constructing the materials, we ensured that the distractor sentence involved the same sense of the preposition as the target sentence (e.g., on to describe support from below and not support via adhesion). We also ensured that the standards did not by themselves give rise to the key spatial semantic category (see Norming Study 2B). The participants in Experiment 1B were instructed to attend carefully to the pictures, whereas the participants in Experiment $1 \mathrm{C}$ were presented with the pictures along with the sentences from Experiment 1A, with the prepositions removed.

The recognition test included all three pictures - the standard, the plus variant, and the minus variant — presented individually in random order. To the extent that language influences recognition memory, we expected to see more plus FAs than minus FAs.

\section{Experiment 1A}

In Experiment 1A, half the participants (the spatial sentences group) received the sentences along with the pictures; the other half (the control group) received only the pictures. Both groups were told to remember the pictures for a later test. If strong linguistic determinism is correctthat is, if the semantic categories of one's language influence cognitive encoding even when language is not being used-we should see more plus FAs than minus FAs for both groups. If the thinking-for-language hypothesis is correct, we should see a plus advantage for the spatial sentences group only. Finally, if there is no effect of language on recognition memory, there should be no difference in the rates of plus and minus FAs for either group.

\section{Method}

Design. Encoding condition (spatial sentences/control), a betweenparticipants factor, was crossed with recognition item type (plus variant/ standard/minus variant), a within-participants factor.

Participants. Thirty-six Northwestern University undergraduates received course credit for their participation in this experiment.

Materials. There were 13 triads of pictures and corresponding sets of sentences as well as 18 filler pictures and sentences. Each triad contained one picture whose figure-ground relation was ambiguous with respect to the preposition in the sentence (the standard), one that fell closer to the center of the spatial category named by the preposition (the plus variant), and one that fell outside of the spatial category 
named by the preposition (the minus variant) (see Figure 1). ${ }^{1}$ All three pictures involved the same objects; the only source of variation was the spatial relation between the two objects. In preparing the pictures, every attempt was made to guard against a possible recognition bias for the plus variant (see the norming studies for Experiment 2). The standard from each triad was used for the study portion of the experiment; all three pictures in the triad were used for the recognition test. For each standard, there was a pair of sentences. One described the figure-ground relation using the key preposition, and the other used the same preposition in the same sense but with two clearly incorrect nouns. The target and distractor sentences are presented in Table 1.

Procedure. The procedure comprised two parts: (1) study and (2) recognition.

Part 1: Study. Twenty-five pictures (13 standards and 12 fillers) were randomized and presented individually for $5 \mathrm{sec}$ each on a computer screen. All participants were told that they would be tested later on their ability to recognize these pictures.

To ensure that the participants in the spatial sentences group processed the sentences, we asked them to choose which of two sentences best described the picture. They were provided with answer sheets with two sentences for each picture: the target sentence and a distractor sentence, as described above. The participants in the control condition were given no additional instructions. After completing the study portion, the participants did an unrelated filler task for $10 \mathrm{~min}$.

Part 2: Recognition. All participants received the same yes/no recognition test. All three of the pictures in each triad were presented individually in random order on a computer screen along with 12 fillers ( 6 old and 6 new), for a total of 51 pictures. The participants were asked to indicate on a numbered answer sheet whether they had seen each picture during the earlier study portion. Each picture remained on the screen until the participant pressed a key to continue.

\section{Results}

The two groups did not differ significantly in their overall error rates, nor in the overall rates of the two kinds of possible errors, misses and FAs (see Figure 2).

The key prediction was that the pattern of FAs would be influenced by the presence of spatial language at study. Indeed, the participants in the spatial sentences condition were significantly more likely to have FAs to the plus variant than to the minus variant (see Figure 3 ) $[t(17)=$ $5.32, p<.0001]$. The participants in the control condition showed no such difference in their FA rate $[t(17)=$ $-0.72, p>.10]$. Thus, having spatial language present at encoding led to a skewing of recognition errors toward the core of the spatial category, providing evidence for an effect of spatial language on the encoding of the pictures.
Discriminability analysis. To further test this claim, we calculated two $d^{\prime}$ measures for each participant: one for the discriminability of the minus variant from the standard and one for the discriminability of the plus variant from the standard. The larger of the two was then determined, ${ }^{2}$ and the participants were pooled by condition. Table 2 shows the results (along with those for Experiments $1 \mathrm{~B}$ and 1C). The control condition showed a symmetric discriminability pattern, with equal numbers of participants showing the two possible discriminability biases. In contrast, the spatial sentences condition showed a strongly skewed discriminability pattern: 12 participants showed greater discriminability for the minus variant, and none showed greater discriminability for the plus variant, a significant difference between conditions $\left[\chi^{2}(2, N=35)=9.65, p<\right.$ $.01]$. As a further check on the effect of condition on discriminability, the set of $d^{\prime}$ data was subjected to a $2 \times 2$ multivariate ANOVA. In support of our conclusion, we found an effect of condition $[F(1,34)=10.72, p=.002]$.

\section{Discussion}

When spatial language was present at encoding, memory for the spatial relations in our pictures was systematically shifted in the direction of the spatial preposition. This is evidence for at least the moderate thinking-for-language version of the Whorfian hypothesis. We argue that this shift reflects an interactive encoding process in which the representation of the pictures is influenced by the spatial sentences. In the next experiment, we asked whether people would spontaneously invoke spatial language when faced with a difficult encoding task. Extrapolating from Brown and Lenneberg's (1954) finding that the codability of colors influenced people's ability to remember them later, we reasoned that if participants believe they are preparing for a difficult memory task, they might mentally invoke linguistic encodings as a memory aid.

\section{Experiment 1B}

In Experiment 1B, we asked whether there are language effects on recognition memory without the overt use of language at encoding. We tested the possibility that participants instructed to pay careful attention to the pictures at study might be induced to encode the pictures linguisti-

Table 1

Sentences Used in Experiments 1 and 2

\begin{tabular}{ll}
\hline \multicolumn{1}{c}{ Target Sentences } & \multicolumn{1}{c}{ Distractor Sentences } \\
\hline The firefly is on the wagon wheel. & The watch is on the nightstand. \\
The puppet is on the table. & The computer is on the desk. \\
The chair is in the corner. & The house is in the middle. \\
The dirt is on the dump truck. & The snow is on the mountain. \\
The ball is under the chair. & The table is under the lamp. \\
The block is on the building. & The plant is on the shelf. \\
The balloon is on the stick. & The kite is on the string. \\
The firefly is in the dish. & The house is in the valley. \\
The coin is in the hand.* & The spaceship is in the crater. \\
The spider is in the bowl of apples. & The shirt is in the basket of laundry. \\
The plane is on the ground. & The woman is on the floor. \\
The hose is around the tree trunk. & The armband is around the arm. \\
The balloon is on the table. & The antenna is on the roof. \\
*Sentences altered for Experiment 2.
\end{tabular}




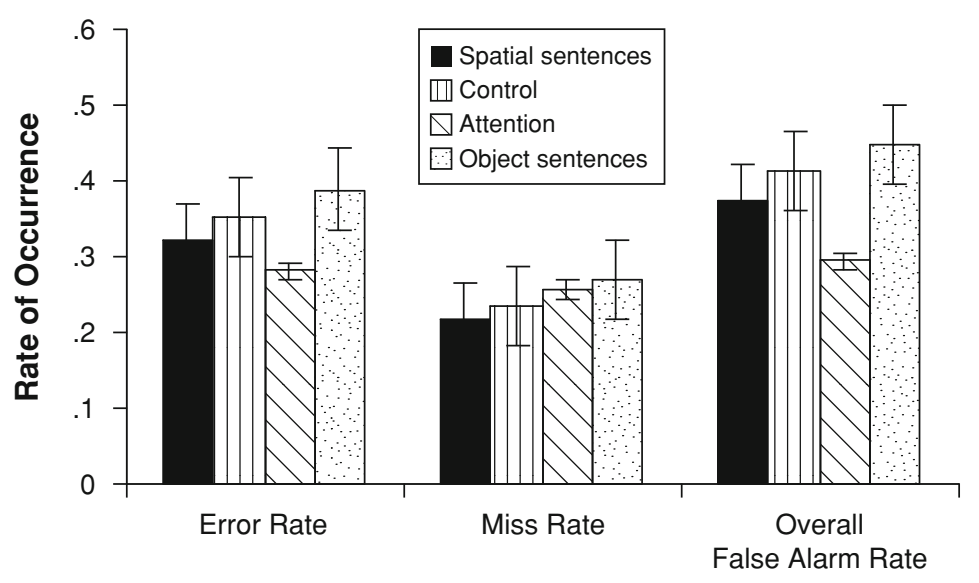

Figure 2. Error rates in Experiments 1A, 1B, and 1C.

cally. If so, they should be more likely to have FAs to the plus variant than to the minus variant, as did the spatial language participants in Experiment 1A.

\section{Method}

Participants. Eighteen Northwestern University undergraduates received course credit for their participation in this experiment.

Materials. The materials were those used in Experiment 1A

Procedure. The procedure was as in the control condition in Experiment 1A, except that the participants were instructed to pay careful attention to the pictures because the recognition test would be very difficult. After completing the study portion, the participants did an unrelated filler task for $10 \mathrm{~min}$. They were then given the same recognition test as in Experiment 1A.

\section{Results and Discussion}

The overall error rate observed in Experiment 1B $(M=$ $.28, S D=.09)$ was lower than that observed in Experiment $1 \mathrm{~A}(M=.34, S D=.08)$, suggesting that the participants did pay more careful attention to the pictures during study (see Figure 2). In particular, the participants in Experiment 1B had a lower incidence of FAs than did the participants in either of the conditions from Experiment 1A [Experiment 1A control, $M=.41, S D=.10$;
Experiment 1A spatial sentences, $M=.38, S D=.08$; Experiment 1B, $M=.29, S D=.08$; comparison with control, $t(34)=3.78, p<.001$; comparison with spatial sentences, $t(34)=2.91, p<.01]$.

However, we found no evidence that the participants in Experiment 1B mentally invoked spatial language as a memory aid. They did not show the error pattern characteristic of spatial language use (more plus than minus FAs). Instead, like the control group in Experiment 1A, the participants in Experiment 1B showed roughly equal numbers of plus and minus FAs $[t(17)=-1.27, p=.22]$.

So far, we have evidence for the influence of spatial language when it is explicitly presented, although not for the stronger possibility that language can affect encoding and recognition when it is not overtly present. In Experiment $1 \mathrm{C}$, we tested the specificity of the language effect. If the recognition shift is due to spatial language, then we should not see this shift if participants are given verbal descriptions that do not contain spatial language.

\section{Experiment 1C}

Experiments 1A and 1B provided evidence for a thinkingfor-language version of the language-and-thought hypoth-

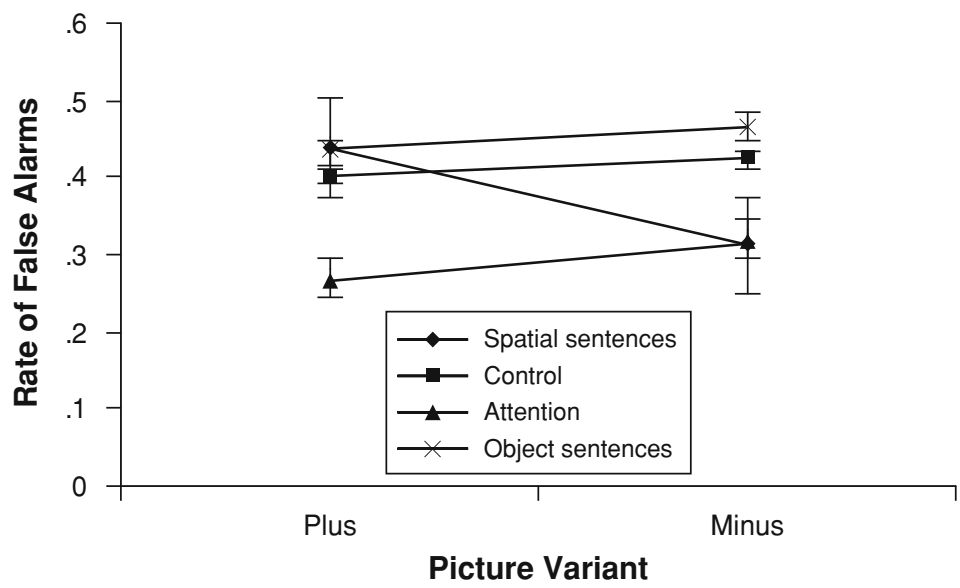

Figure 3. False alarms by condition in Experiments 1A, 1B, and 1C. 
Table 2

Participants Pooled According to the Discriminability Analysis in Experiments 1A, 1B, and 1C

\begin{tabular}{lccc}
\hline \multicolumn{1}{c}{ Group } & $\begin{array}{c}d^{\prime} \text { Larger } \\
\text { for Plus }\end{array}$ & $\begin{array}{c}d^{\prime} \text { Larger } \\
\text { for Minus }\end{array}$ & $\begin{array}{c}d^{\prime} \\
\text { Equal }\end{array}$ \\
\hline Control & 4 & 4 & 10 \\
Spatial sentences & 0 & 12 & 5 \\
Attention* $^{*}$ & 8 & 4 & 5 \\
Object sentences $^{\dagger}$ & 6 & 3 & 7 \\
\hline
\end{tabular}

* One participant was dropped from the analysis due to a hit rate of 1 . †One participant was dropped from the analysis due to a hit rate of 1 , and another was dropped due to a plus false alarm rate of 0 .

esis: that using spatial language during the encoding of scenes fosters spatial representations congruent with the language. In Experiment 1C, we investigated the reverse possibility: that using language that emphasizes only the objects present (i.e., eliminating the spatial language) will invite encodings that are primarily centered on the objects. Therefore, participants given object-centered language will experience no shift toward the core of the spatial category (and thus will not show any differential tendency to have FAs to the plus variants over the minus variants). Furthermore, if language about the objects induces object-centered encodings, then we might find an overall loss of accuracy in the ability to retain the spatial relations, leading to depressed performance on the recognition task (in which participants must reject alternatives that share objects but differ slightly in their spatial relations). Although this outcome seems most plausible, there is another possibility: It could be that the presence of any linguistic stimuli at encoding will entrain participants into a linguistic mode and prompt them to think of the semantic category that best fits the standard. If the presence of language induces a general mind-set to invoke applicable semantic encodings, participants given language at encoding (even nonspatial language) should show a greater rate of plus FAs than minus FAs.

We tested these hypotheses by presenting the participants with sentences during encoding that simply pointed out the objects without spatial prepositions. Equal plus and minus FAs in this case would support a semantically specific version of the thinking-for-language hypothesis, whereas a prevalence of plus FAs would support a more general effect of language.

\section{Method}

Participants. Eighteen Northwestern University undergraduates received course credit for their participation in this experiment.

Materials. The pictures were the same as those in Experiment 1A. We modified the sentences on the participants' answer sheets from those used in Experiment 1A by removing the prepositions (e.g., "The picture shows a block and a building" and "The picture shows a plant and a shelf").

Procedure. The procedure was identical to that in the spatial sentences condition in Experiment 1A: The participants saw pictures and circled the appropriate sentence; they then did a 10-min filler task and, finally, were given a recognition test.

\section{Results and Discussion}

The participants who were given object sentences at encoding made more errors than did those told to pay close attention (Experiment 1B) or those given spatial sentences (Experiment 1A); in particular, they were more likely to have FAs than were the participants in the attention condition (see Figure 2). This recognition disadvantage was confirmed by independent samples $t$ tests [total errors for spatial vs. object sentences, $t(34)=-2.04, p<.05$; total errors for attention vs. object sentences, $t(34)=-3.35, p<.005$; FAs for attention vs. object sentences, $t(34)=-3.82, p<.001]$.

However, object sentence participants failed to show any recognition shift toward the core of the spatial category designated by the preposition. They demonstrated equal plus and minus FAs $[M=.43(S D=.18)$ and $M=$ $.47(S D=.15)$, respectively; $t(17)=-0.94, p=.36]$, much as did the no-language participants in the previous experiments. This pattern differed from the pattern shown by spatial sentences participants in Experiment 1A. This provides support for the suggestion that the spatial preposition was specifically responsible for the pattern of responses observed in the language condition in Experiment 1A, a thinking-for-language result. The complete FA breakdown for Experiment 1 is presented in Figure 3.

Discriminability analysis. In order to compare across the four conditions, two $d^{\prime}$ measures were calculated for each individual participant in Experiments 1B and 1C (as in Experiment 1A): one for the discriminability of the minus variant from the standard, and one for that of the plus variant from the standard. The larger of the two was then determined, and the participants were pooled by condition (see Table 2). For both the attention group (Experiment 1B) and the object sentences group (Experiment $1 \mathrm{C}$ ), we found that the participants more often failed to discriminate the minus variant (which was designed to be perceptually more similar to the standard) than the plus variant; that is, the minus variant was more confusable with the standard than was the plus variant. This contrasts with the pattern found for the spatial sentences group (Experiment 1A), for which the plus variant was more confusable with the standard than was the minus variant. This difference in discriminability between conditions was significant $\left[\chi^{2}(6, N=68)=19.31, p<.01\right]$.

As another comparison across the four conditions, we conducted a 4 (condition) $\times 2$ ( $d^{\prime}$ type: plus or minus) ANOVA over the $d^{\prime}$ data. As predicted, there was an interaction between condition and $d^{\prime}$ type $[F(3,64)=5.67, p=$ $.002]$. Post hoc $t$ tests revealed that the standard was more confusable with the plus variant than with the minus variant for the spatial sentences group $[t(16)=-5.52, p<.0001$ by a Bonferroni correction]; the other three conditions showed no difference. The interaction of condition by $d^{\prime}$ type did not reach significance in the items analysis $[F(3,36)=2.34$, $p=.09]$. However, $t$ tests showed the same pattern as for the participants analysis: the standard was more confusable with the plus variant than with the minus variant for the spatial sentences group only $[t(7)=-4.81, p=.002]$.

\section{EXPERIMENT 2}

In Experiment 1, we found that recognition memory for spatial scenes was influenced by spatial language presented at study. These results provide support for the interactive 
encoding claim that the participants adjusted their encoding of the picture to better accord with the spatial preposition.

However, in Experiment 1, the participants received all three recognition items (standard, plus, and minus) for each triad (randomly ordered) in the yes/no recognition task. This leaves open the possibility that responses to a given item might have been influenced by having seen other items from the same triad during the recognition task. In Experiment 2, we changed the design of the recognition test to eliminate this possibility. We replicated the basic plan of Experiment 1A, but, in the recognition test, we presented each participant with only one recognition item from each triad. In addition, to ensure that the materials met the requirement of the design, we performed three norming studies.

\section{Method}

Design. The factors were encoding condition (spatial sentences/ control; between participants), recognition item type (plus variant/ standard/minus variant; within participants), and assignment condition (between participants).

Participants. One hundred eighteen Northwestern University undergraduates received course credit for their participation. An additional 86 Northwestern University undergraduates participated in the norming studies.

Materials. We preformed three norming studies on the materials used in Experiment 1. In Norming Study 2A, we asked 24 Northwestern University undergraduates to rate the applicability of the spatial sentences to each of the items in order to verify that the spatial sentences were most applicable to the plus variants and least applicable to the minus variants, with intermediate applicability to the standard pictures. We found that, as expected, participants gave the highest ratings to the plus variants $(M=5.72, S D=$ $.70)$, followed by the standards $(M=4.47, S D=1.16)$, and then by the minus variants $(M=2.54, S D=1.63)[F(2,36)=22.35, p<$ $.0001]$. However, examination of the results for individual triads showed a different pattern for two of the triads: one depicting a coin in a hand, and one depicting a firefly in a dish. These sentences were adjusted accordingly for Experiment 2 . The changed sentences are indicated with asterisks in Table 1.

In Norming Study 2B, we collected free descriptions of the standards (from 12 Northwestern University undergraduates) and plus variants (from 10 Northwestern University undergraduates) in order to check whether (1) the plus variants would elicit the target spatial prepositions from Experiment $1 \mathrm{~A}$ and (2) the standards would be less likely to elicit consistent spatial prepositions, indicating that they were indeed ambiguous with respect to the spatial sentences. As expected, the spontaneous descriptions varied considerably, particularly for the standards. The rate of use of the key spatial prepositions was .25 in descriptions of the standards $(S D=.25)$. Also as expected, there was a trend for the key prepositions to be used more often in descriptions of the plus variants $(M=.32, S D=.32)$, although this difference did not reach significance $[t(12)=-1.01, p>.10]$.

In Norming Study 2C, we collected ratings of the degree of similarity between the plus variant and the standard and between the minus variant and the standard from 40 Northwestern University undergraduates to verify that both variants were equally similar to the standard. We were especially concerned to rule out an imbalance in similarity in favor of the plus variant, since this could provide an alternate explanation for the plus FAs. Although the overall ratings were comparable for the two alternatives, participants gave slightly higher similarity ratings to the minus variants $(M=4.96, S D=$ 1.40) than to the plus variants $(M=4.73, S D=1.51)[t(39)=$ $-0.47, p<.0001]$. Across individual triads, the minus variant was judged more similar to the standard for five triads, the plus variant was judged more similar for three triads, and they were judged equally similar for five triads.
As a result of the norming studies, we made minor modifications to two of the triads of pictures for use in Experiment $2,{ }^{3}$ and a change of preposition (from in to on) in the sentences corresponding to two others (indicated with asterisks in Table 1). One triad (depicting a balloon on a stick) was eliminated so that the number of triads would be divisible by three as required by the design.

Procedure. The study procedure was identical to that of Experiment $1 \mathrm{~A}$. Also as before, both conditions received the same yes/no recognition test after a 10-min filled delay. However, each participant saw only one picture from each triad at recognition (instead of all three as in Experiment 1). These were presented in random order along with 12 fillers ( 6 old and 6 new) on a computer screen. As in Experiment 1, the participants were asked to indicate on a printed answer sheet whether they had seen each picture during the earlier study portion. Each picture remained on the screen until the participant pressed a key.

\section{Results}

As in Experiment 1A, we found the predicted effect of spatial language. The participants in the spatial sentences condition were significantly more likely to have FAs to the plus variant than to the minus variant, whereas participants in the control condition showed no such asymmetry (Figure 4). The difference between the plus FAs and the minus FAs was significant only in the spatial sentences condition $[t(57)=6.87, p<.0001]$. In addition, the difference in the rate of FAs between the two groups only reached significance for the responses to the plus variant $[t(116)=-3.93, p=.0001]$.

Also as in Experiment 1A, we found no difference in the miss rates between the control and spatial sentences groups $[t(116)=1.46, p>.10]$. In contrast to Experiment $1 \mathrm{~A}$, the overall FA rates for the two groups in Experiment 2 did differ (Figure 5), with FAs being more prevalent among the participants in the spatial sentences condition $[t(116)=$ $-1.77, p<.05$, one-tailed]. As the analysis of the pattern of FAs (above) shows, this difference was entirely attributable to a difference in responses to the plus variants.

Discriminability analysis. As in Experiment 1, two $d^{\prime}$ measures were calculated for each individual participant - one for minus and one for plus. The larger of the two was then determined, and the participants were pooled by condition (see Table 3 ).

The results of the $d^{\prime}$ analysis for Experiment 2 replicate those for Experiment 1. In the spatial sentences condition, the dominant pattern was one of greater discriminability between the standard and the minus variant than between the standard and the plus variant. However, in the control condition, an equal number of participants had a larger minus $d^{\prime}$ as had a larger plus $d^{\prime}$, a significant difference between conditions $\left[\chi^{2}(2, N=118)=16.67, p<.0001\right]$. As a further check on the effect of condition on discriminability, the set of $d^{\prime}$ data was subjected to a $2 \times 2$ multivariate ANOVA. In support of our conclusion, we found an effect of condition $[F(2,115)=6.63, p=.002]$.

\section{EXPERIMENT 3}

Our experiments so far have provided evidence for the hypothesis that language interacts with visual input to influence the encoding of pictures. In Experiment 2, as in Experiment 1, the recognition results showed a shift 


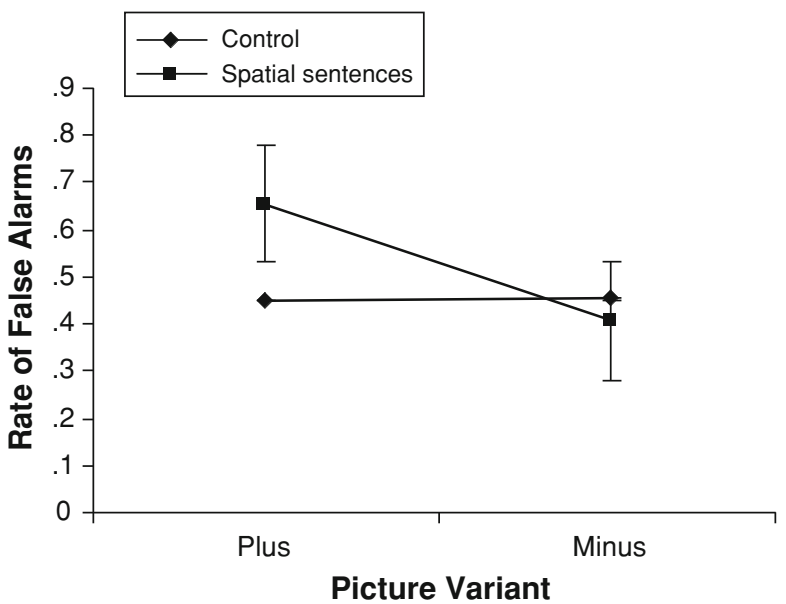

Figure 4. False alarms by condition in Experiment 2.

toward the core of the spatial category when spatial language was present at encoding, consistent with the interactive encoding account.

However, before drawing strong conclusions, we need to address a possible alternate explanation for these effects, which we term the separate encoding account. Because the effect of language in Experiments 1 and 2 was to shift recognition in the direction of the specific terms present at encoding, it could be that the effect was simply due to memory for the language presented, rather than to differential encoding of the pictures. This line of reasoning has been articulated most clearly in research on memory overlay effects, including effects of verbal questioning on eyewitness testimony (Loftus \& Palmer, 1974; McCloskey \& Zaragoza, 1985) and effects of language (Carmichael, Hogan, \& Walter, 1932) and of verbal overshadowing (Schooler \& Engstler-Schooler, 1990) on the reproduction of visual materials. In this arena, the issue of whether language alters prior visual memories or merely exists as an alternate, possibly more available encoding has been the source of much debate. In Loftus and Palmer's classic study, participants viewed a filmed automobile accident, then answered questions about what they had viewed. The results showed that the phrasing of the questions (e.g., use of the verb smash rather than the verb collide) influenced the likelihood that participants would falsely claim to have seen a given element (e.g., broken glass), suggesting that the subsequent language had altered participants' memorial representation of the event. However, McCloskey and Zaragoza argued that the results could have come about without a direct effect of verbal information on pictorial memory. To demonstrate this, they presented participants with a scene that included Target Object A and then referred to it as Object B in postevent questioning. At test, participants were asked to determine whether they had seen Object A, which was present in the scene, or Object $\mathrm{C}$, which was neither present in the scene nor mentioned in postevent discussion. They found that misleading information did not result in lower accuracy in this task (in contrast to the case when A was contrasted with $\mathrm{B}$ at test), from which they concluded that the verbal information had not changed participants' memory for the original event. As this research shows, finding effects of language on retrieval of visually presented information does not necessarily imply effects of language on the visual representation itself. Such effects could also arise from two separately encoded and stored memory traces, if participants draw on their verbal memory to supplement their visual memory. On this account, the results of Experiments 1 and 2 (i.e., the elevation in plus FAs) would be explained by people resorting to their stored language trace when their memory for a picture is weak, rather than by an interactive encoding process by which language influences the encoding of the pictures.

One important difference from the present research is that McCloskey and Zaragoza's (1985) discussion was directed at studies in which the visual materials were encoded first, with verbal descriptions following later. Thus, they asked whether later verbal input can alter a prior visual memory, whereas, in our experiments, we tested for interactions of verbal and visual materials presented together. In other words, we are arguing for an interactive encoding process, as opposed to the retroactive alteration process that was the focus of McCloskey and Zaragoza's critique. But obviously, the separate encoding explanation could still apply.

Experiment 3 was designed with two goals in mind: to test whether our language effect could be accounted for

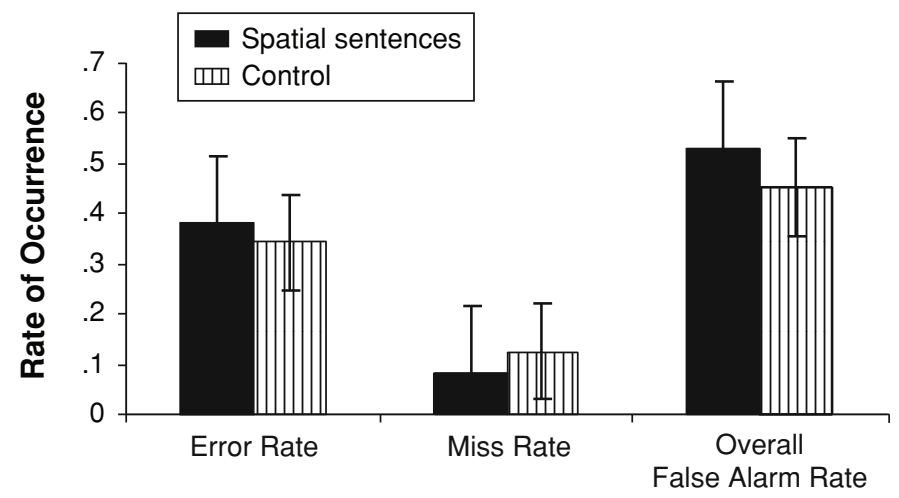

Figure 5. Error rates in Experiment 2. 
Table 3

Participants Pooled According to the Discriminability Analysis in Experiment 2

\begin{tabular}{lccc}
\hline Group & $\begin{array}{c}d^{\prime} \text { Larger } \\
\text { for Plus }\end{array}$ & $\begin{array}{c}d^{\prime} \text { Larger } \\
\text { for Minus }\end{array}$ & $\begin{array}{c}d^{\prime} \\
\text { Equal }\end{array}$ \\
\hline Control & 20 & 20 & 20 \\
Spatial sentences & 4 & 38 & 16 \\
\hline
\end{tabular}

by the separate encoding possibility and (if possible) to extend the scope of the language effect. Happily, there is a way to accomplish both these goals at once. Rather than testing for narrow, direct effects of language (i.e., whether participants' picture encodings shift in the direction of the specific terms used), we tested for a more global effect, using a task inspired by McCloskey and Zaragoza's (1985) technique. We asked whether participants will show indirect effects of using nonappropriate spatial terms. Specifically, in Experiment 3, we addressed the question of whether introducing a nonappropriate spatial term will call attention to spatial semantics more generally and lead to a shift toward the more appropriate spatial category.

\section{Experiment 3A}

In Experiment $3 \mathrm{~A}$, the participants saw the standard pictures immediately preceded by sentences. Half the sentences were the key preposition sentences of the earlier experiments. The other half were clearly not appropriate - that is, they expressed a very different spatial relation from the one that best fit the scene. For example, for the standard in Figure 1, the key preposition sentence (the plausible description) was "The block is on the building" and the nonappropriate preposition sentence was "The block is in the building." The participants were told to read the sentences and to remember the pictures for a later recognition task. (Obviously, given this instruction, the participants could have ignored the sentences; in Experiment 3B, we verified that they did not.) They were then given a two-alternative forced choice (2AFC) recognition test between the actually presented standard and the plus variant (incorrect). On the basis of our prior results, for pictures that were coupled with the key preposition, we expected the participants to (incorrectly) choose the plus variant. The question was what will happen with the nonappropriate prepositions?

Suppose that the interactive encoding account is correct and, further, that hearing a spatial term calls forth not just the specific category associated with the term but, more generally, the semantic set or dimension it belongs to. Then, hearing a clearly nonappropriate spatial preposition may prompt participants to consider which spatial preposition should apply. That is, participants may think (for example) "This isn't in, it's on." They will substitute the correct (or most applicable) spatial relation-which, by design, is the key prepositional category. If this reasoning holds, the effect of being given a nonappropriate preposition should be (paradoxically) to increase FAs to the plus variants.

In contrast, the separate encoding account predicts the pattern found by McCloskey and Zaragoza (1985): no rise in FAs for pictures paired with the nonappropriate preposition. The nonappropriate-preposition sentences do not match either of the recognition pictures. Thus, if participants encode the sentences and pictures separately and, during test, resort to the sentences when they forget the pictures (as in the separate encoding account), then for the nonappropriate preposition sentences, their sentence memory will be irrelevant to the choice between the standard and the plus variant. They should therefore show no elevation in FAs.

Thus, both accounts predict that participants will often have FAs to the plus variant when the picture has been coupled with the key preposition; but they make different predictions for pictures coupled with the nonappropriate prepositions. The interactive encoding account predicts a high FA rate for pictures presented with either the key prepositions or the nonappropriate prepositions. The separate encoding account predicts a high FA rate only for pictures presented with the key prepositions.

\section{Method}

Participants. Twenty Northwestern University undergraduates received course credit for their participation.

Design and Materials. The factors were encoding condition (key preposition/nonappropriate preposition, within participants) and assignment condition (between participants). Each participant saw 12 standards and 8 fillers - a total of 20 trials, of which half contained key-preposition sentences and half contained nonappropriatepreposition sentences.

The pictures and the key-preposition sentences were as in Experiment 2 . The nonappropriate-preposition sentences were created from the key-preposition sentences by replacing the preposition with another that was incompatible with both the standard picture and the plus variant. In selecting these, we avoided nonappropriate prepositions that could name the minus variant. For example, for the block and the building depicted in Figure 1, in which the plus FA is on and the minus FA is off, we used in as the nonappropriate preposition. The complete set of sentences is presented in Table 4.

Procedure. The procedure comprised two parts: (1) study and (2) recognition.

Part 1: Study. The pictures were presented individually for $3 \mathrm{sec}$ each on a computer screen in randomized order. Each picture was preceded by a sentence, which was displayed for $1 \mathrm{sec}$. The participants were told that they would read sentences followed by corresponding pictures and that they would later be asked to recognize only the pictures. For half of the pictures, the key spatial sentence (from Experiment 2) was presented; for the other half, a nonappropriatepreposition sentence was displayed. For the filler pictures, we used incorrect sentences that had the right preposition but the wrong noun (i.e., the distractor sentences from Experiment 1A). This was done to keep the participants from noticing a pattern that the incorrect sentences always erred in their spatial prepositions.

Part 2: Recognition. After a 10-min filled delay, all participants received the same $2 \mathrm{AFC}$ recognition test. The participants were shown each standard paired with its plus variant and asked to click on the picture they had seen during the study portion. The pictures remained on the screen until the participant made a choice.

\section{Results and Discussion}

The key prediction of the separate encoding account is that the FA rate will be higher for pictures that were coupled with key spatial prepositions than for those coupled with nonappropriate prepositions. In fact, there was a nonsignificant trend in the reverse direction [key preposi- 
tions, $M=.12, S D=.17$; nonappropriate prepositions, $M=.16, S D=.15 ; t(19)=.79, p>.4]$. Thus, the results argue against the possibility that the language effects in Experiments 1 and 2 were simply due to use of independently encoded key-preposition sentences and buttress the interactive encoding account of the language effect.

In addition, the results extend the interactive encoding account beyond purely local effects of words as category attractors. In Experiment $3 \mathrm{~A}$, hearing nonappropriate spatial prepositions apparently led to an indirect language effect wherein the participants shifted to the most appropriate spatial category, thus achieving a common construal of sentence and picture.

\section{Experiment 3B}

Experiment $3 \mathrm{~B}$ was a manipulation check, conducted to verify that the participants did indeed attend to the sentences. To do this, we gave 20 new participants the same materials as in Experiment 3A, but we tested memory for sentences instead of memory for the pictures. The study phase was exactly as in Experiment 3A, including the instruction to remember only the pictures for a recognition test. However, after a $10 \mathrm{~min}$ filled interval, we administered a surprise sentence recognition test. Because we wanted to test for memory of all the sentences presented, we used a yes/no recognition test rather than a forced choice task. Each participant was given a packet containing one sentence per study item, and responded "yes" or "no" to the question "Did this sentence appear in part 1?" Half the sentences were old (i.e., seen during the study phase), and half were new. Within each class, half contained the key preposition, and half contained the nonappropriate preposition.

\section{Results}

The results showed high sentence recognition accuracy (mean proportion correct $=.83, S D=.16$ ), allowing us to rule out the possibility that the sentences were ignored. Memory for the key-preposition sentences $(M=.88, S D=$ .16) was nonsignificantly better than memory for the nonappropriate-preposition sentences $(M=.78, S D=.13)$. Thus, the failure to find a difference in the FA rates for the two encoding conditions in Experiment 3A was not due to the participants' having failed to process the sentences.
Interestingly, we also found evidence that interactive encoding works both ways. The participants were more likely to misrecognize the sentence when a nonappropriate preposition had been seen at study $(M=.28, S D=$ $.27)$ than when a key preposition had been seen $(M=.08$, $S D=.21)[t(19)=2.26, p<.05]$, suggesting that the sentences were adjusted to fit the pictures as well as the pictures to fit the sentences.

\section{Discussion}

According to interactive encoding, people's encoding of pictures can be influenced by accompanying language. This hypothesis received initial support from our finding that picture recognition was influenced by the presence of spatial language during encoding in Experiments 1 and 2. In Experiment 3A, we found evidence that language effects can extend beyond the application of specific categories. Our results suggest that the language effect may often be to focus attention on a particular dimension, rather than to impose the stated value on that dimension.

In this experiment, we also tested a possible alternate explanation for the effect of language - that the pictures and sentences were encoded separately and that the effect of language on recognition resulted from the participants' consulting their sentence memory when pictorial memory was weak. Contrary to the prediction of the separate encoding account (but consistent with the interactive encoding account), there was no difference in FA rates as a function of sentence type; indeed, there was a nonsignificant imbalance in the opposite direction. The results of Experiment $3 \mathrm{~B}$ verified that the sentences were processed and retained. The results further showed that sentences that were inconsistent with the pictures (those with nonappropriate prepositions) were more likely to be altered than those that were more consistent with the pictures. Both these findings are in line with the interactive encoding hypothesis.

We further examined the data across all three experiments for item effects. However, we found no systematic pattern across experiments as to which items showed the greatest tendency for picture memory to be affected by verbal labels, nor for this effect to correlate (or anticorrelate) with the degree to which sentence memory was affected by the spatial scenes.

Table 4

Sentences Used in Experiment 3

\begin{tabular}{ll}
\hline \multicolumn{1}{c}{ Key-Preposition Sentences } & Nonappropriate-Preposition Sentences \\
\hline The firefly is on the wagon wheel. & The firefly is under the wagon wheel. \\
The puppet is on the table. & The puppet is under the table. \\
The chair is in the corner. & The chair is below the corner. \\
The dirt is on the dump truck. & The dirt is under the dump truck. \\
The ball is under the chair. & The ball is on the chair. \\
The block is on the building. & The block is in the building. \\
The firefly is on the dish. & The firefly is under the dish. \\
The coin is on the hand. & The coin is under the hand. \\
The spider is in the bowl of apples. & The spider is under the bowl of apples. \\
The plane is on the ground. & The plane is beneath the ground. \\
The hose is around the tree trunk. & The hose is beneath the tree trunk. \\
The balloon is on the table. & The balloon is below the table. \\
\hline
\end{tabular}


The results help refine and extend the thinking-forlanguage account. The effect of language in our experiments was to focus attention on the spatial relations and to alter both the encoding of the scene and the encoding of the sentence in the direction of the most consistent prepositional category. When a linguistic assertion disagrees with the perceptual facts, the effect can be to move the encodings to a different value within the same semantic set - one in which the language and the scene can agree.

\section{GENERAL DISCUSSION}

The results of our experiments provide evidence that spatial language can influence the way people encode and remember spatial relations in visual scenes. Our standards were designed to be unlikely members of the key spatial category (indeed, the spatial preposition was rarely used in spontaneous descriptions; see Norming Study 2B). However, the participants given spatial prepositions during encoding of the standards showed a shift in picture recognition toward the core of the spatial category denoted by the preposition (Experiments 1A and 2). That is, they were significantly more likely to have FAs to pictures closer to the center of the prepositional category than to those less central. The participants who received the pictures without spatial sentences at study showed no such shift, even when they were asked to pay close attention to the pictures; their FA rates were symmetrical (Experiments 1B and 2). Finally, in Experiments $3 \mathrm{~A}$ and 3B, we ruled out the possibility that these language effects simply reflect the use of a separately encoded verbal memory, rather than an interactive encoding of sentence and picture. Contrary to the separate encoding prediction, the misrecognition effect was not restricted to the category directly named in the sentence. Rather, we found that hearing even a nonappropriate spatial preposition induced a shift toward the key-preposition category. This finding is incompatible with the use of an independently encoded verbal store. The pattern suggests an interaction between language and perception during encoding.

These experiments link two bodies of work that have largely been considered independently. One is the neoWhorfian investigations on effects of language on perceptual and conceptual representation and reasoning reviewed in the introduction. The other is research on memory overlay effects. In the latter arena, the issue of whether language alters prior visual memories or merely exists as an alternate, possibly more available encoding has been the source of much debate. The results of our experiments show that when spatial language is presented simultaneously (or just prior) to visual materials, the interaction between linguistic categories and visual materials can influence the encoding of spatial relations in both the pictures and the sentences.

Not all spatial tasks are susceptible to language effects. For example, Huttenlocher and her colleagues used a location reproduction task (Crawford, Regier, \& Huttenlocher, 2000; Huttenlocher, Hedges, \& Duncan, 1991), in which participants saw a dot and a quadrilateral reference object; the dot (but not the quadrilateral) then disappeared, and participants indicated where on the screen the dot had been. The participants' errors were skewed toward the center of an inferred geometric category, even after rating the applicability of a (different) linguistic category to the array. One difference between the two studies is that, in the Huttenlocher et al. studies, memory was probed immediately after the disappearance of the dot, whereas we imposed a 10-min delay. This delay might have contributed to the language effect. In addition, we speculate that the language effects may be more likely with naturalistic materials such as those used in our task than with simple geometric objects such as those used by Huttenlocher and her colleagues.

Our finding of an interaction between verbal and visual encodings can also be related to recent accounts of language comprehension that propose that sentences are understood by constructing perceptual simulations of the events described (Barsalou, 1999; Glenberg, 1997; Zwaan, 2004). Such accounts propose a close interaction between perceptual and semantic processing. For example, Zwaan and Yaxley (2003) found that spatial iconicity affects semantic relatedness judgments (e.g., people were faster to judge BASEMENT and ATTIC as semantically related words when they saw ATTIC above BASEMENT than when they saw BASEMENT above ATTIC). Zwaan, Madden, Yaxley, and Aveyard (2004) have also shown effects of language on perceptual judgments. For example, participants were shown pictures of objects and asked to judge whether the second object was the same as the first. Before each picture pair, participants heard sentences such as "You tossed the beach ball over the sand toward the kids" or "The kids tossed the beach ball over the sand toward you." Responses were faster when the meaning of the sentence matched the size difference of the objects. That is, if the sentence described a ball moving toward the viewer, participants were faster to respond when the second picture showed a ball that was larger than the first. The authors suggest that, in this case, the thoughts triggered by the sentence were compatible with the size discrepancy between the balls, allowing a fast response. They concluded that "words can, indeed, move mental representations."

According to this view, we activate positional and orientational information when presented with linguistic stimuli (e.g., Zwaan et al., 2004; Zwaan \& Yaxley, 2003). In the case of spatial prepositions, this information might correspond to core members of the spatial prepositional categories, leading to the observed shift in picture representation toward the center of the prepositional category when a spatial preposition has been processed during encoding.

To return to the neo-Whorfian literature, our findings are most compatible with a thinking-for-language hypothesis (see Slobin, 1996), whereby language influences thought when one is either producing or comprehending language. Across our studies, we saw no evidence that people engaged in the covert use of language to encode the pictures, even when they were told to pay close attention. Of course, our design, with its deliberately ambiguous standards, might have underestimated the effects of language. The low rate of spontaneous use of the key 
prepositions in the free description task (Norming Study 2B) suggests that even if the participants did encode the pictures linguistically in the attention task, they were unlikely to have used our spatial sentences. ${ }^{4}$ However, overall, we have evidence for language effects only when language is present.

\section{Extended Effects}

We found that processing a clearly nonappropriate preposition just prior to a scene led to a kind of contrast effect: The participants encoded the scene in terms of a competing (and more applicable) spatial prepositional category. This suggests an indirect effect of language, rather than a simple direct substitution of the named concept into the scene. Here, the use of a linguistic term appears to call forth a system of semantic categories, from which participants choose the most appropriate member. This effect is both more subtle and potentially more pervasive than a simple direct insertion effect.

However, these results invite further questions. Why did we find indirect effects of extraneous language when McCloskey and Zaragoza (1985) did not? (Recall that McCloskey \& Zaragoza found that retroactive false information concerning a presented object did not impair participants' ability to correctly recognize the object they had viewed, as opposed to a new, previously unmentioned, object.) One possibility is that language effects - both direct insertion effects and indirect semantic system effects - are more likely when language is presented before or during the encoding of the perceptual materials (as in our experiments) than when the language occurs after the perceptual materials have been encoded (as in McCloskey \& Zaragoza's experiments). This would be consistent with the interactive encoding account.

Another intriguing possibility is that a further contributor to the difference between the two studies is the kinds of items used. McCloskey and Zaragoza (1985) used object substitution (e.g., they showed a hammer and referred verbally to a screwdriver). In contrast, our manipulation involved a change of spatial relation. There is reason to believe that relational terms such as prepositions (and many verbs and adjectives) are more likely to belong to systems of interrelated semantic categories than are concrete nouns (Gentner \& Boroditsky, 2001; Huttenlocher \& Lui, 1979). It seems plausible that people are more likely to see such interrelated categories as contrastive and to shift from one to another. We might therefore expect the extended effects explored here to be strongest for relational terms and to be much weaker (if they occur at all) for object terms. A question for future research is whether and how the technique explored here would apply across different kinds of semantic systems.

Finally, we note that the meanings of spatial relational terms incorporate nonspatial factors such as support and containment relations, intended function, and so on (Bowerman \& Pederson, 1992; Coventry \& Garrod, 2004; Coventry, Prat-Sala, \& Richards, 2001; Feist, 2000, 2004; Feist \& Gentner, 2003; Talmy, 1988; Vandeloise, 1991), in addition to geometry. Given that spatial meaning is in fact quite complex, a question for further research is how these factors interact with geometric factors in the encoding of spatial scenes.

\section{Thinking for Language}

This work fits with recent discussions that have made finer distinctions within the language-and-thought arena. Gentner and Goldin-Meadow (2003) distinguish three versions of the claim that language influences thought: language as lens, language as tool kit, and language as category shaper. The language-as-lens view is the strong "Whorfian hypothesis" of linguistic determinism: that the language we acquire determines how we perceive and represent the world. On the language-as-category-shaper view, human categories are relatively universal, but language can influence the boundaries (Papafragou et al., 2002), implying enduring, if limited, effects on cognition. We espouse the language-as-tool-kit view: that acquiring a language provides new representational resources that augment the capacity for encoding and reasoning (Dennett, 1993; Gentner, 2003; Vygotsky, 1934/1962). On this view, language provides tools that facilitate forming and holding particular construals, but it does not replace all other encoding formats (Gentner \& Goldin-Meadow, 2003).

Slobin's (1996) thinking-for-speaking (or more broadly, thinking-for-language) hypothesis is essentially orthogonal to these distinctions, as it concerns when language effects occur rather than what the effects are. However, it is most compatible with the second and third versions of the language-and-thought hypothesis given above. When conversing, people are particularly likely (1) to encode and reason using the semantic tools provided by their language and (2) to honor the semantic distinctions of the language. Our findings are compatible with such a thinking-for-language account, although of course they do not rule out stronger effects of language in other contexts (see Haun, Rapold, Call, Janzen, \& Levinson, 2006; Majid et al., 2004). Our findings here are less dramatic than those predicted by the strong Whorfian hypothesis, but they still leave room for pervasive semantic effects, given the prominence of language in human mental life. As Pinker (1989, p. 360) states, "Whorf was surely wrong when he said that one's language determines how one conceptualizes reality in general. But he was probably correct in a much weaker sense: one's language does determine how one must conceptualize reality when one has to talk about it."

\section{AUTHOR NOTE}

This work was supported by NSF-LIS Award SBR-9720313 and NSFROLE Award 21002/REC-0087516 and was completed while the first author was a postdoctoral fellow at Northwestern University. We thank Kathleen Braun and Michelle Osmondson for help in conducting and analyzing the study, Jonathan Cohen and Benjamin Scott-Hopkins for help in preparing the manuscript, and Beth Levin, Jason Jameson, Jeff Loewenstein, and Phillip Wolff for helpful discussions of the ideas and methods. We also thank Satoru Suzuki for the $d^{\prime}$ analysis program, Jeff Rouder for discussions of the statistical analyses, and Melissa Bowerman for allowing us access to her materials. Finally, we thank Karen Emmorey, Nora Newcombe, Rolf Zwaan, and an anonymous reviewer for helpful comments on earlier versions of this study. Correspondence 
concerning this article should be addressed to M. I. Feist, University of Louisiana, Institute of Cognitive Science, P.O. Drawer 43772, Lafayette, LA 70504-3772 (e-mail: feist@louisiana.edu).

Note-This article was accepted by the previous editorial team, when Colin M. MacLeod was Editor.

\section{REFERENCES}

Barsalou, L. W. (1999). Perceptual symbol systems. Behavioral \& Brain Sciences, 22, 577-660.

Billman, D., \& KRYCH, M. (1998). Path and manner verbs in action: Effects of "skipping" and "exiting" on event memory. In Proceedings of the 20th Annual Conference of the Cognitive Science Society (pp. 156-161). Mahwah, NJ: Erlbaum.

BORODITSKY, L. (2001). Does language shape thought? English and Mandarin speakers' conceptions of time. Cognitive Psychology, 43, 1-22.

Bowerman, M., \& Choi, S. (2003). Space under construction: Language-specific spatial categorization in first language acquisition. In D. Gentner \& S. Goldin-Meadow (Eds.), Language in mind: Advances in the study of language and cognition (pp. 387-427). Cambridge, MA: MIT Press.

Bowerman, M., \& Levinson, S. C. (EDs.) (2001). Language acquisition and conceptual development. Cambridge: Cambridge University Press.

Bowerman, M., \& Pederson, E. (1992, December). Crosslinguistic perspectives on topological spatial relationships. Paper presented at the 91st Annual Meeting of the American Anthropological Association, San Francisco.

Bowerman, M., \& Pederson, E. (1996). Crosslinguistic perspectives on topological spatial relationships. Manuscript in preparation.

Brown, R. W., \& Lenneberg, E. H. (1954). A study in language and cognition. Journal of Abnormal \& Social Psychology, 49, 454-462.

Carmichael, L., Hogan, H. P., \& Walter, A. A. (1932). An experimental study of the effect of language on the reproduction of visually perceived form. Journal of Experimental Psychology, 15, 73-86.

Cheng, K., \& Newcombe, N. S. (2005). Is there a geometric module for spatial orientation? Squaring theory and evidence. Psychonomic Bulletin \& Review, 12, 1-23.

ChoI, S., \& Bowerman, M. (1991). Learning to express motion events in English and Korean: The influence of language-specific lexicalization patterns. Cognition, 41, 83-121.

Coventry, K. R., Carmichael, R., \& Garrod, S. C. (1994). Spatial prepositions, object-specific function, and task requirements. Journal of Semantics, 11, 289-309.

Coventry, K. R., \& Garrod, S. C. (2004). Saying, seeing, and acting: The psychological semantics of spatial prepositions. New York: Psychology Press.

Coventry, K. R., Prat-Sala, M., \& Richards, L. (2001). The interplay between geometry and function in the comprehension of over, under, above, and below. Journal of Memory \& Language, 44, 376-398.

Crawford, L. E., Regier, T., \& Huttenlocher, J. (2000). Linguistic and non-linguistic spatial categorization. Cognition, 75, 209-235.

DennetT, D. C. (1993). Learning and labeling. Mind \& Language, 8 , 540-548.

FeIst, M. I. (2000). On in and on: An investigation into the linguistic encoding of spatial scenes. Unpublished doctoral dissertation, Northwestern University, Evanston, IL.

FeIst, M. I. (2004, August). Talking about space: A cross-linguistic perspective. Paper presented at the 26th Annual Meeting of the Cognitive Science Society, Chicago.

Feist, M. [I.], \& Gentner, D. (2003, August). Factors involved in the use of in and on. Paper presented at the 25th Annual Meeting of the Cognitive Science Society, Boston

Gennari, S. P., Sloman, S. A., Malt, B. C., \& Fitch, W. T. (2002). Motion events in language and cognition. Cognition, 83, 49-79.

Gentner, D. (2003). Why we're so smart. In D. Gentner \& S. GoldinMeadow (Eds.), Language in mind: Advances in the study of language and thought (pp. 195-235). Cambridge, MA: MIT Press.

Gentner, D., \& Boroditsky, L. (2001). Individuation, relativity, and early word learning. In M. Bowerman \& S. C. Levinson (Eds.), Language acquisition and conceptual development (pp. 215-256). Cambridge: Cambridge University Press.
Gentner, D., \& Goldin-Meadow, S. (Eds.) (2003). Language in mind: Advances in the study of language and thought. Cambridge, MA: MIT Press.

GentNer, D., \& ImaI, M. (1992). Is the future always ahead? Evidence for system-mappings in understanding space-time metaphors. In Proceedings of the 14th Annual Meeting of the Cognitive Science Society (pp. 510-515). Hillsdale, NJ: Erlbaum.

Gentner, D., Imai, M., \& Boroditsky, L. (2002). As time goes by: Evidence for two systems in processing space-time metaphors. Language \& Cognitive Processes, 17, 537-565.

GentNer, D., \& Loftus, E. F. (1979). Integration of verbal and visual information as evidenced by distortions in picture memory. American Journal of Psychology, 92, 363-375.

Glenberg, A. M. (1997). What memory is for. Behavioral \& Brain Sciences, 20, 1-55.

Gumperz, J. J., \& Levinson, S. C. (EDs.) (1996). Rethinking linguistic relativity. Cambridge: Cambridge University Press.

Haun, D. B. M., Rapold, C. J., Call, J., Janzen, G., \& Levinson, S. C. (2006). Cognitive cladistics and cultural override in hominid spatial cognition. Proceedings of the National Academy of Sciences, 103, 17568-17573.

Hermer-Vazquez, L., Moffet, A., \& Munkholm, P. (2001). Language, space, and the development of cognitive flexibility in humans: The case of two spatial memory tasks. Cognition, 79, 263-299.

Hermer-Vazquez, L., Spelke, E. S., \& Katsnelson, A. S. (1999). Sources of flexibility in human cognition: Dual-task studies of space and language. Cognitive Psychology, 39, 3-36.

Hunt, E., \& Agnoli, F. (1991). The Whorfian hypothesis: A cognitive psychology perspective. Psychological Review, 98, 377-389.

Huttenlocher, J., Hedges, L. V., \& Duncan, S. (1991). Categories and particulars: Prototype effects in estimating spatial location. Psychological Review, 98, 352-376.

HutTenlocher, J., \& Lui, F. (1979). The semantic organization of some simple nouns and verbs. Journal of Verbal Learning \& Verbal Behavior, 18, 141-162.

Learmonth, A. E., Nadel, L., \& Newcombe, N. S. (2002). Children's use of landmarks: Implications for modularity theory. Psychological Science, 13, 337-341.

Levinson, S. C. (1996). Frames of reference and Molyneux's question: Crosslinguistic evidence. In P. Bloom, M. A. Peterson, L. Nadel, \& M. F. Garrett (Eds.), Language and space (pp. 109-169). Cambridge, MA: MIT Press.

Levinson, S. [C.] (2003). Language and mind: Let's get the issues straight. In D. Gentner \& S. Goldin-Meadow (Eds.), Language in mind: Advances in the study of language and cognition (pp. 25-46). Cambridge, MA: MIT Press.

Levinson, S. C., Kita, S., Haun, D. B. M., \& Rasch, B. H. (2002). Returning the tables: Language affects spatial reasoning. Cognition, 84, $155-188$

Levinson, S. [C.], Meira, S., \& the Language and Cognition Group (2003). "Natural concepts" in the spatial topological domainAdpositional meanings in crosslinguistic perspective: An exercise in semantic typology. Language, 79, 485-516.

Li, P., \& Gleitman, L. (2002). Turning the tables: Language and spatial reasoning. Cognition, 83, 265-295.

LoEWENSTEIN, J., \& GeNTNER, D. (2001). Spatial mapping in preschoolers: Close comparisons facilitate far mappings. Journal of Cognition \& Development, 2, 189-219.

Loewenstein, J., \& Gentner, D. (2005). Relational language and the development of relational mapping. Cognitive Psychology, 50, 315-353.

Loftus, E. F., \& PALMER, J. C. (1974). Reconstruction of automobile destruction: An example of the interaction between language and memory. Journal of Verbal Learning \& Verbal Behavior, 13, 585-589.

LuCY, J. A. (1992). Grammatical categories and cognition: A case study of the linguistic relativity hypothesis. Cambridge: Cambridge University Press.

Majid, A., Bowerman, M., Kita, S., Haun, D. B. M., \& Levinson, S. C. (2004). Can language restructure cognition? The case for space. Trends in Cognitive Sciences, 8, 108-114.

Malt, B. C., Sloman, S. A., \& Gennari, S. (2003). Speaking vs. thinking about objects and actions. In D. Gentner \& S. Goldin-Meadow (Eds.), Language in mind: Advances in the study of language and thought (pp. 81-111). Cambridge, MA: MIT Press. 
McCloskey, M., \& Zaragoza, M. (1985). Misleading postevent information and memory for events: Arguments and evidence against memory impairment hypotheses. Journal of Experimental Psychology: General, 114, 1-16.

Papafragou, A., Massey, C., \& Gleitman, L. (2002). Shake, rattle, 'n' roll: The representation of motion in language and cognition. Cognition, 84, 189-219.

Pederson, E., Danziger, E., Wilkins, D., Levinson, S. C., Kita, S. \& Senft, G. (1998). Semantic typology and spatial conceptualization. Language, 74, 557-589.

PINKER, S. (1989). Learnability and cognition: The acquisition of argument structure. Cambridge, MA: MIT Press.

REgIER, T. (1996). The human semantic potential: Spatial language and constrained connectionism. Cambridge, MA: MIT Press.

SAPIR, E. (1929). The status of linguistics as a science. Language, $\mathbf{5}$, 207-214.

Schooler, J. W., \& Engstler-Schooler, T. Y. (1990). Verbal overshadowing of visual memories: Some things are better left unsaid. Cognitive Psychology, 22, 36-71.

SLobIN, D. I. (1996). From "thought and language" to "thinking for speaking.” In J. J. Gumperz \& S. C. Levinson (Eds.), Rethinking linguistic relativity (pp. 70-96). Cambridge: Cambridge University Press.

Slobin, D. I. (2003). Language and thought online: Cognitive consequences of linguistic relativity. In D. Gentner \& S. Goldin-Meadow (Eds.), Language in mind: Advances in the study of language and cognition (pp. 157-191). Cambridge, MA: MIT Press.

TAlmy, L. (1988). Force dynamics in language and cognition. Cognitive Science, 12, 49-100.

TAYlor, H. A., \& TVersky, B. (1996). Perspective in spatial descriptions. Journal of Memory \& Language, 35, 371-391.

VANDEloise, C. (1991). Spatial prepositions: A case study from French (A. R. K. Bosch, Trans.). Chicago: University of Chicago Press.
Vygotsky, L. S. (1962). Thought and language. Cambridge, MA: MIT Press. (Original work published 1934)

WHORF, B. L. (1956). Language, thought, and reality: Selected writings of Benjamin Lee Whorf (J. B. Carroll, Ed.). Cambridge, MA: MIT Press.

ZWAAN, R. A. (2004). The immersed experiencer: Toward an embodied theory of language comprehension. In B. H. Ross (Ed.), The psychology of learning and motivation (Vol. 44, pp. 35-62). New York: Academic Press.

ZwaAn, R. A., Madden, C. J., Yaxley, R. H., \& Aveyard, M. E. (2004) Moving words: Dynamic representations in language comprehension. Cognitive Science, 28, 611-619.

ZwaAn, R. A., \& Yaxley, R. H. (2003). Spatial iconicity affects semantic relatedness judgments. Psychonomic Bulletin \& Review, 10, 954-958.

\section{NOTES}

1. Three of the triads (those depicting a ball under a chair, a chair in a corner, and a hose around a tree trunk) were adapted from drawings in Melissa Bowerman's topological picture series.

2. All $d^{\prime}$ measures within .25 of one another were considered equal.

3 . For the triad depicting a firefly and a wagon wheel, the firefly was changed to make its wings visible, making it appear to be flying, the wood grain on the wheel was changed to be more realistic, and the background color was changed from gray to light blue. For the triad depicting a chair and a corner, the lengths of the walls were adjusted to be equal in all three pictures, and the distance between the chair and the corner was increased in the standard and minus variant, making the pictures more discriminable.

4. We thank an anonymous reviewer for suggesting this possibility.

(Manuscript received October 1, 2004; revision accepted for publication July 26, 2005.) 